\title{
Isolation and Localization of Mesenchymal Stem Cells in Human Palatine Tonsil by W5C5 (SUSD2)
}

\author{
Byung-Joo Lee ${ }^{\mathrm{a}}$ Dae-Woon Kang ${ }^{\mathrm{a}}$ Hee-Young Park ${ }^{\mathrm{a}}$ Ji-Sun Song ${ }^{\mathrm{a}}$ Ji-Min Kim ${ }^{\mathrm{a}}$ \\ Jeon-Yeob Jang ${ }^{a}$ Jin-Choon Lee ${ }^{b}$ Soo-Geun Wang ${ }^{a}$ Jin Sup Jung ${ }^{c}$ \\ Sung-Chan Shin ${ }^{\mathrm{a}}$
}

\begin{abstract}
aDepartment of Otorhinolaryngology-Head and Neck Surgery, Pusan National University School of Medicine and Biomedical Research Institute, Pusan National University, Busan, ${ }^{b}$ Department of Otorhinolaryngology-Head and Neck Surgery, Pusan National University School of Medicine and Biomedical Research Institute, Pusan National University Yangsan Hospital, Yangsan, Kyeongnam, 'Department of Physiology, School of Medicine, Pusan National University, Yangsan, Korea
\end{abstract}

\section{Key Words}

Mesenchymal stem cells $\bullet$ Tonsil $\cdot$ W5C5 $・$ SUSD2 $・$ Localization

\begin{abstract}
Background/Aims: Although tonsil-mesenchymal stem cells (T-MSCs) have been studied as a new autologous or homologous source of MSCs, research on specific markers of MSCs and localization for purified T-MSC isolation has not yet been reported. This study investigates the expression of W5C5 (SUSD2) in tonsil stromal cells and the colony-forming ability and differentiation potential of $\mathrm{W} 5 \mathrm{C} 5+$ cells to determine the usefulness of $\mathrm{W} 5 \mathrm{C} 5+\mathrm{MSCs}$ as a marker that can be used for the purification of T-MSCs. In addition, the location of W5C5+ cells expressed in the tonsil tissues is examined. Methods: T-MSCs were isolated from the tonsillar tissues of 12 patients undergoing tonsillectomy. The colony-forming ability, surface markers, proliferation potential, and differentiation capacities of purified W5C5+ MSCs, W5C5- MSCs, and unselected T-MSCs were evaluated. The location of the W5C5+ cells in the tonsillar tissues was also investigated by immunohistochemistry. Results: W5C5 was expressed in $2.5 \pm 0.4 \%$ of fresh human tonsil stromal cells. W5C5+ cells formed many colonies, but W5C5- cells did not form any colonies. The colony-forming number of W5C $5+$ cells $(74.4 \pm 9.8)$ was significantly higher than that of unselected tonsil stromal cells $(23.6 \pm 3.7)$. However, the differences in proliferation potential, surface marker expression, and differentiation potential between W5C5 + T-MSCs and unselected T-MSCs were not significant. W5C5+ cells were identified in the perivascular area around the blood vessels. Conclusion: W5C5+ T-MSCs possessed typical MSC properties with high colony-forming efficiency, and niches of W5C5+ T-MSCs were located in the perivascular area of tonsil tissues. These findings suggest that W5C5 is a useful single marker for the isolation of purified T-MSCs.
\end{abstract}




\section{Introduction}

Mesenchymal stem cells (MSCs) are multipotent cells that are able to self-renew and differentiate into several cell types of the same mesenchymal lineage, including osteocytes, adipocytes, and chondrocytes [1-3]. MSCs are ideal candidates for cell-based therapy and regenerative medicine because of their self-renewal abilities and their multipotent and paracrine effects $[4,5]$. Although bone marrow (BM) is a good source of MSCs, BM-MSCs have some limitations in terms their harvest and yield in cell-based therapy and regenerative medicine applications. Recent research has identified alternative sources of MSCs, including adipose, placental, dental pulp, endometrial, umbilical cord, palatine tonsil, and adenoid tissue [6-12]. An MSC source that is obtainable in large quantities under local anesthesia with minimal discomfort would be ideal.

Conventional cultured MSCs represent a heterogeneous population of cells $[13,14]$. Plastic-adherent cells obtained from tissues or organs contain a number of other cells types that have the ability to adhere to a plastic surface, including endothelial cells, fibroblasts, and monocytes. Conventional in vitro methods for MSC isolation have a major disadvantage due to the contamination with these cells. For future tissue engineering or cell-based therapies to become a reality, the identification of stem cell-specific markers for their prospective isolation is crucial. Although recent studies have sought to identify specific MSC markers for prospective isolation in several MSCs, there is no consensus on a single surface marker for identifying MSCs [13].

The human palatine tonsil is readily accessible to otolaryngologists and easily obtained by tonsillectomy or punch biopsy. Recent studies reported the isolation of tonsilMSCs (T-MSCs) from tonsil tissues and found that the morphology, cell-surface markers, proliferation potential, and differentiation potential of T-MSCs were similar to those of BMMSCs $[11,15]$. T-MSCs were considered to be promising MSCs that could be used as future alternative sources for autologous or allogenic MSCs, because the effects of donor age, longterm passage culture, and cryopreservation on T-MSC properties were negligible $[11,15]$. Despite the reports of T-MSCs as a new autologous or homologous source of MSCs, there have been no reports on specific markers of MSCs for purified T-MSC isolation. In addition, tonsil tissue is composed of the tonsil epithelium of endodermal origin and lymphoid tissue of mesodermal origin. Although T-MSCs can be isolated in tonsil tissues of two different origins, there have been no reports on the location of isolated T-MSCs. The cultures from several stromal tissues are heterogeneous, comprising stromal, endothelial, and perivascular cells, and are not particularly enriched for MSCs. The advantage of using specific surface markers for isolation of MSCs is that enriched clonogenic and self-renewing MSCs can easily obtain. Several cell-surface markers have been used to enrich MSCs from various tissue sources. W5C5, a known epitope of the sushi domain containing two (SUSD2) molecules, was recently identified as a novel marker for endometrial MSCs [16].

W5C5 has been introduced as a novel marker for the prospective isolation of highly clonogenic endometrial MSCs that were able to reconstitute endometrial stromal tissues in vivo $[3,9,17]$. However, there have been no reports on the expression and functional differences of W5C5 in MSCs derived from tonsil tissues. Our study aimed to investigate the expression of W5C5 in tonsil stromal cells and the colony-forming ability and differentiation potential of W5C5+ cells to determine the usefulness of W5C5+ MSCs as a marker that can be used for the purification of T-MSCs. In addition, the location of W5C5+ cells expressed in the tonsil tissues was examined.

\section{Materials and Methods}

Human palatine tonsil tissues and isolation of tonsil stromal cells

Tonsils were obtained after obtaining informed consent from 12 patients ( 5 - 15 years old) undergoing tonsillectomy because of chronic tonsillar hypertrophy and/or chronic tonsillitis. Before surgery, all research 


\section{Cellular Physiology Cell Physiol Biochem 2016;38:83-93 \begin{tabular}{l|l|l|} 
and BOI: 10.1159/000438611 & $\begin{array}{l}\text { C) 2016 The Author(s). Published by S. Karger AG, Basel } \\
\text { www.karger.com/cpb }\end{array}$ \\
\hline Published online: January 08, 2016
\end{tabular}}

Lee et al.: Isolation and Localization of T-MSCs by W5C5

subjects provided their informed written consent to use of their tissue specimens for research purposes. Our institutional review board approved the protocol.

Tonsil stromal cells were isolated as described previously. Briefly, to isolate palatine tonsil stromal cells, tonsil tissues samples were washed with phosphate-buffered saline (PBS) and digested at $37^{\circ} \mathrm{C}$ for 30 min with $0.075 \%$ type I collagenase. The enzyme activity was neutralized with $\alpha$-modified Eagle's medium $(\alpha-$ MEM) containing $10 \%$ fetal bovine serum (FBS). The dissociated cells were filtered through a sterile $70-$ um cell strainer. Most of the stromal cells and blood cells passed through the cell strainer into a sterile tube, whereas the undigested fragments were retained on the strainer.

Tonsil stromal W5C5+ cell sorting and culture

Flow cytometric analysis was used to isolate W5C5+ MSCs. At least 50,000 isolated tonsil stromal cells (in $100 \mu \mathrm{LBS} / 0.5 \%$ bovine serum albumin (BSA) $/ 2 \mathrm{mmol} / \mathrm{L}$ EDTA) were incubated with FITC-conjugated monoclonal antibodies against human W5C5 (BioLegend, San Diego, CA. USA). Cells were then washed and resuspended in 1uM SYTOX Blue to distinguish live and dead cells; fluorescence-activated cell sorting (FACS) was undertaken on a FACSAria II (BD Biosciences, San Diego, CA).

Freshly sorted cells were cultured and incubated overnight at $37^{\circ} \mathrm{C}$ under $5 \% \mathrm{CO}_{2}$ in a control medium ( $\alpha$-MEM, $10 \%$ FBS, $100 \mathrm{U} / \mathrm{ml}$ of penicillin, and $100 \mu \mathrm{g} / \mathrm{ml}$ of streptomycin). Following incubation, the tissue culture plates were washed to remove any residual non-adherent cells and then maintained at $37^{\circ} \mathrm{C}$ under $5 \% \mathrm{CO}_{2}$ in the control medium. One week later, when a monolayer of adherent cells had reached confluency, cells were trypsinized (0.05\% trypsin-EDTA; Sigma), resuspended into the media, and subcultured at a concentration of 2,000 cells $/ \mathrm{cm}^{2}$. Cells were subcultured every 4-5 days.

For colony-forming assays for passage 0 cells, W5C5+ cells, W5C5- cells, and unselected cells were plated on $1 \times 10^{5} / 60-\mathrm{cm}^{2}$ dishes and cultured for 7 days. The dishes were stained with $0.5 \%$ crystal violet, and the colonies were counted. Colonies less than $2 \mathrm{~mm}$ in diameter and faintly stained colonies were ignored.

\section{Cell proliferation assays}

Cell proliferation was evaluated by an MTT assay. W5C5+ and unselected T-MSCs were plated at a density of $1 \times 10^{4}$ cells/well in 24-well plates and evaluated after 1, 2, 3 and 4 days of culture. After washing the cells, a culture medium containing $0.5 \mathrm{mg} / \mathrm{ml}$ of MTT was added to each well. Cells were incubated for $2 \mathrm{~h}$ at $37^{\circ} \mathrm{C}$, the supernatant was removed, and the formazan crystals that formed in viable cells were solubilized with $200 \mu \mathrm{l}$ of dimethyl sulfoxide. A 100- $\mu \mathrm{l}$ aliquot of each sample was then transferred to 96well plates, and the absorbance of each well was measured at $560 \mathrm{~nm}$ with an ELISA Reader equipped with XFLUOR4 software (version 4.51). This experiment was repeated four times. For the colony-forming unit (CFU) assay for passage 2 cells, W5C5+ and unselected T-MSCs were plated at a density of 100 cells in 60$\mathrm{mm}$ plates and allowed to grow for 7 days. The cultures were terminated and stained with crystal violet for colony visualization. The colonies were counted manually under an inverted microscope.

\section{Flow cytometric analysis}

Flow cytometric analysis was used to characterize the phenotypes of isolated W5C5+ and unselected MSCs. Isolated W5C5+ and unselected MSCs (in $100 \mu \mathrm{L} \mathrm{PBS/0.5 \%} \mathrm{BSA/2} \mathrm{mmol/L} \mathrm{EDTA)} \mathrm{were} \mathrm{incubated}$ with FITC-conjugated monoclonal antibodies against CD45, CD31, HLA-DRY, CD90, CD44, and CD73 (BD Biosciences, Durham, NC, USA). Labeled cells were analyzed by flow cytometry using a FACS Caliber flow cytometer equipped with Cell Quest Pro software (BD Biosciences).

Differentiation potential of tonsil W5C5+ and unselected T-MSCs

Adipogenic differentiation. Adipogenic differentiation was induced by culturing W5C5+ and unselected T-MSCs for 3 weeks in adipogenic media ( $\alpha$-MEM supplemented with 10\% FBS, 1 uM dexamethasone, 100 $\mu \mathrm{g} / \mathrm{ml}$ 3-isobutyl-1 methylxanthine, $5 \mu \mathrm{g} / \mathrm{mL}$ insulin, and $60 \mu \mathrm{M}$ indomethacin) and assessed by oil red 0 (Sigma) staining as an indicator of intracellular lipid accumulation. Prior to staining, the cells were fixed for $15 \mathrm{~min}$ at room temperature in 70\% ethanol. Cells were then incubated in $2 \%$ oil red 0 reagent for $1 \mathrm{~h}$ at room temperature. Excess stain was removed by washing with $70 \%$ ethanol and then distilled water to visualize lipid droplets.

Osteogenic differentiation: Osteogenic differentiation was induced by culturing W5C $5+$ and unselected 


\section{Cellular Physiology Cell Physiol Biochem 2016;38:83-93

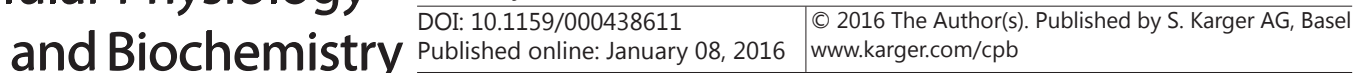

Lee et al.: Isolation and Localization of T-MSCs by W5C5

T-MSCs for 3 weeks in osteogenic media ( $\alpha$-MEM supplemented with $10 \%$ FBS, $0.1 \mathrm{mM}$ dexamethasone, $10 \mu \mathrm{M} \beta$-glycerophosphate, and $50 \mu \mathrm{g} / \mathrm{mL}$ ascorbic acid) followed by examining extracellular matrix calcification by alizarin red S (Sigma) staining. For alizarin red S staining, cells were fixed with $70 \%$ ethanol, washed with distilled water, incubated in $2 \%$ alizarin red solution for $15 \mathrm{~min}$ at room temperature, and finally washed numerous times with distilled water.

Chondrogenic differentiation. Chondrogenic differentiation was induced using the micromass culture technique. Briefly, $10 \mu \mathrm{L}$ of a concentrated W5C5+ and unselected MSC suspension $\left(3 \times 10^{5}\right.$ cells $\left./ \mathrm{mL}\right)$ was plated onto the center of each well and allowed to attach at $37^{\circ} \mathrm{C}$ for $2 \mathrm{~h}$. Chondrogenic media ( $\alpha$-MEM supplemented with $1 \% \mathrm{FBS}, 0.1 \mathrm{mM}$ dexamethasone (Sigma), $50 \mu \mathrm{g} / \mathrm{mL}$ ascorbic acid, insulin-transferrinselenium (ITS+1; Sigma), and $10 \mathrm{ng} / \mathrm{mL}$ TGF- $\beta 1$ (Sigma)) was gently overlaid to avoid detaching the cell nodules, and the culture was maintained in chondrogenic media for 4 weeks before analysis. Chondrogenesis was confirmed by immunohistochemistry for collagen type-II staining. For collagen type-II $\alpha 1$ staining, sections were blocked with $10 \%$ horse serum, incubated with purified anti-mouse collagen type-II $\alpha 1$ antibody (BD Bioscience) for $1 \mathrm{~h}$, and washed with PBS (pH 7.4). Antibody-bound cells were detected with a peroxidase substrate kit (VECTASTAIN ABC Kit; Vector Laboratories). Sections were washed, counterstained with hematoxylin, and examined by light microscopy.

Real-time polymerase chain reaction (PCR)

Total cellular RNA was isolated from the cells and reverse transcribed using conventional protocols. The primer sequences used in the experiment were as follows: glyceraldehyde-3-phosphate dehydrogenase (GAPDH), 5' - GGA CTC ATG ACC ACA GTC CAT GCC - 3', 5' - TCA GGG ATG ACC TTG CCC ACA - 3'; lipoprotein lipase (LPL), 5'- GAG ATT TCT CTG TAT GGC ACC- $3^{\prime}, 5^{\prime}$ - CTG CAA ATG AGA CAC TTT CTC - $3^{\prime}$; peroxisome proliferator-activated receptor-gamma (PPAR $\gamma$ ), 5'- TGA ATG TGA AGC CCA TTGAA - $3^{\prime}, 5^{\prime}$-CTG CAG TAG CTG CAC GTGTT -3'; adipogenesis, alkaline phosphatase (ALP), 5'- TGG AGC TTC AGA AGC TCA ACA CCA -3', 5' - ATC TCG TTG TCT GAG TAC CAG TCC -3'; osteocalcin (OC), 5' - ATG AGA GCC CTC ACA CTC CTC - $3^{\prime}, 5^{\prime}$ GCC GTA GAA GCG CCG ATA GGC - $3^{\prime}$; collagen type II $\alpha 1$ (COL2A1), $5^{\prime}$ - GGA AAC TTT GCT GCC CAG ATG - $3^{\prime}$, 5'- TCA CCA GGT TCA CCA GGA TTGC -3'; aggrecan (AGN), 5'- TGC GGG TCA ACA GTG CCT ATC - -3', 5'- CAC GAT GCC TTT CAC CAC GAC - $3^{\prime}$. All of the primer sequences were determined using established GenBank sequences. Real-time quantitation was based on the LightCycler assay, using a fluorogenic SYBR Green I reaction mixture for PCR with a LightCycler instrument (Roche, Mannheim, Germany). The amplification program consisted of one cycle of $95^{\circ} \mathrm{C}$ with a 60 -s hold ("hot start"), followed by 40 cycles of $95^{\circ} \mathrm{C}$ with a 0 -s hold, a specified annealing temperature with a 5 -s hold, $72^{\circ} \mathrm{C}$ with a 12 -s hold, and a specified acquisition temperature with a 2-s hold. All experiments were conducted three times, and negative and positive controls were included in all experiments. GAPDH mRNA was amplified as an internal control. LightCycler software, version 3.3 (Roche Diagnostics), was used to analyze PCR kinetics and calculate quantitative data. A standard curve generated in a separate run was loaded into runs of each sample (without standard curves). Each run included one sample of known concentration in the range covered by the standard curve, thus allowing the estimation of exact copy numbers by the second derivative maximum method. For each sample, copy numbers of target gene mRNA were divided by those of GAPDH mRNA to normalize for target gene mRNA expression, thus avoiding sample-to-sample differences in RNA quantity.

\section{Immunocytochemistry and immunohistochemistry}

Cells were fixed with 4\% paraformaldehyde for 10 min, permeabilized with $0.5 \%$ Tween 20 for 10 $\mathrm{min}$, and blocked in 1\% BSA for $1 \mathrm{~h}$ at room temperature. Fixed cells were stained with purified rabbit anti-human W5C5 for $2 \mathrm{~h}$ at room temperature and incubated with PE-conjugated-goat anti-rabbit IgG (e-Bioscience) for $1 \mathrm{~h}$ at room temperature. Cells were mounted with DAPI containing the VECTASHIELD mounting medium and examined using a fluorescence microscope.

Paraffin sections were prepared from human tonsil tissues. The sections were deparaffinized, washed three times in PBS, and transferred to a blocking buffer (PBS containing 2\% BSA) for $1 \mathrm{~h}$ at room temperature prior to application of the primary antibody. The sections were stained with purified rabbit anti-human W5C5 in combination with purified mouse anti-human CD31 overnight at $4^{\circ} \mathrm{C}$. Cells were incubated with PE-conjugated-goat anti-rabbit IgG (e-Bioscience) and FITC-conjugated-goat anti-mouse IgG (e-Bioscience) for $1 \mathrm{~h}$ at room temperature. Cells were mounted with DAPI containing the VECTASHIELD mounting medium and examined using a confocal laser-scanning microscope. 
Statistical analyses

Student's t-test or the Mann-Whitney test was performed to determine the statistical significance between the two groups. Data are presented as means \pm SEM for parametric data and medians and ranges for nonparametric data. Results were considered statistically significant when $\mathrm{p}<0.05$.

\section{Results}

Isolation and CFU assay of W5C5+ cells from human tonsil stromal cells

The expression of W5C5 was examined on fresh tonsil stromal cells by flow cytometry. Tonsil stromal cells were FACS sorted based on positive or negative W5C5 expression. W5C5 was expressed in $2.5 \pm 0.4 \%(n=6)$ of fresh human tonsil stromal cells (Fig. 1-A). We evaluated the clonogenicity of passage 0 cells using a CFU assay. W5C5+ cells formed many colonies, but W5C5- cells did not form any colonies. The colony-forming number of W5C5+ cells $(74.4 \pm 9.8)$ was significantly higher than that of unselected tonsil stromal cells (23.6 \pm 3.7$)$ (Fig. 1-B and C). These results suggest that the W5C5+ population includes most clonogenic cells in human tonsil tissues and that W5C 5 can be used for isolating clonogenic tonsil stromal cells.

To evaluate the proliferative potential in W5C5+ cells and unselected tonsil stromal cells, we determined the number of CFUs after seeding 100 cells. The numbers of CFUs of W5C5+ cells $(81.2 \pm 2.1)$ and unselected cells $(79.4 \pm 1.7)$ did not differ significantly (Fig. 2-A and $\mathrm{B})$. However, the proliferative abilities of W5C5+ cells and unselected cells were similar in the MTT assay (Fig. 2-C).

Surface phenotype of human palatine tonsil W5C5+ cells and unselected cells

There were no significant morphological differences between W5C5+ cells and unselected cells. W5C5+ cells were examined for the expression of lineage markers and

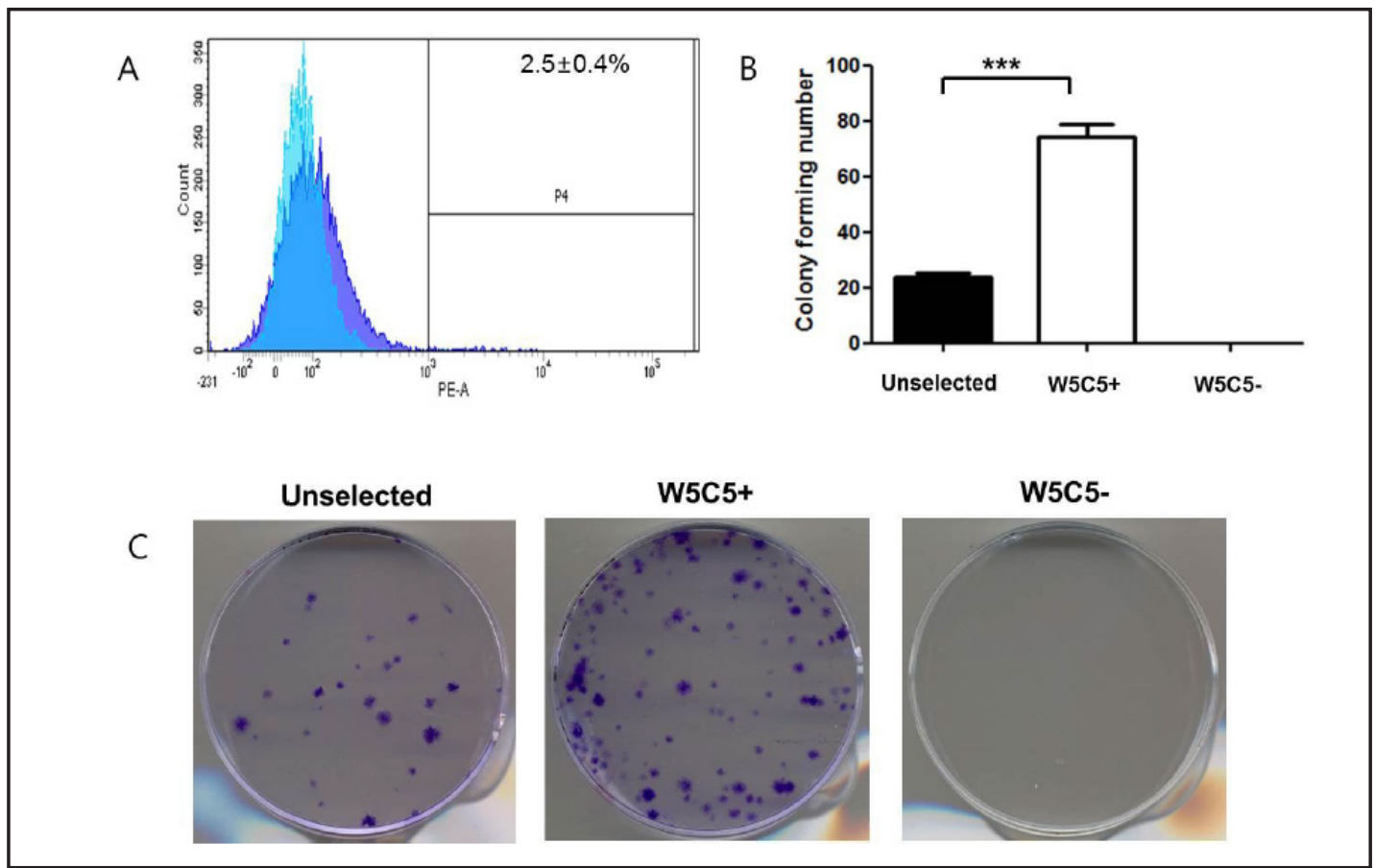

Fig. 1. Isolation and CFU assay of W5C5+ cells from human tonsil stromal cells. (A) FACS results for W5C5 in tonsil stromal cells. W5C5 was expressed in $2.5 \pm 0.4 \%$ of fresh human tonsil stromal cells. (B and C) CFU assay for W5C5+ cells, W5C5- cells, and unselected T-MSCs. W5C5+ cells formed many colonies, but W5C5cells did not form any colonies. The colony-forming number of W5C5+ cells was significantly higher than that of unselected tonsil stromal cells. 


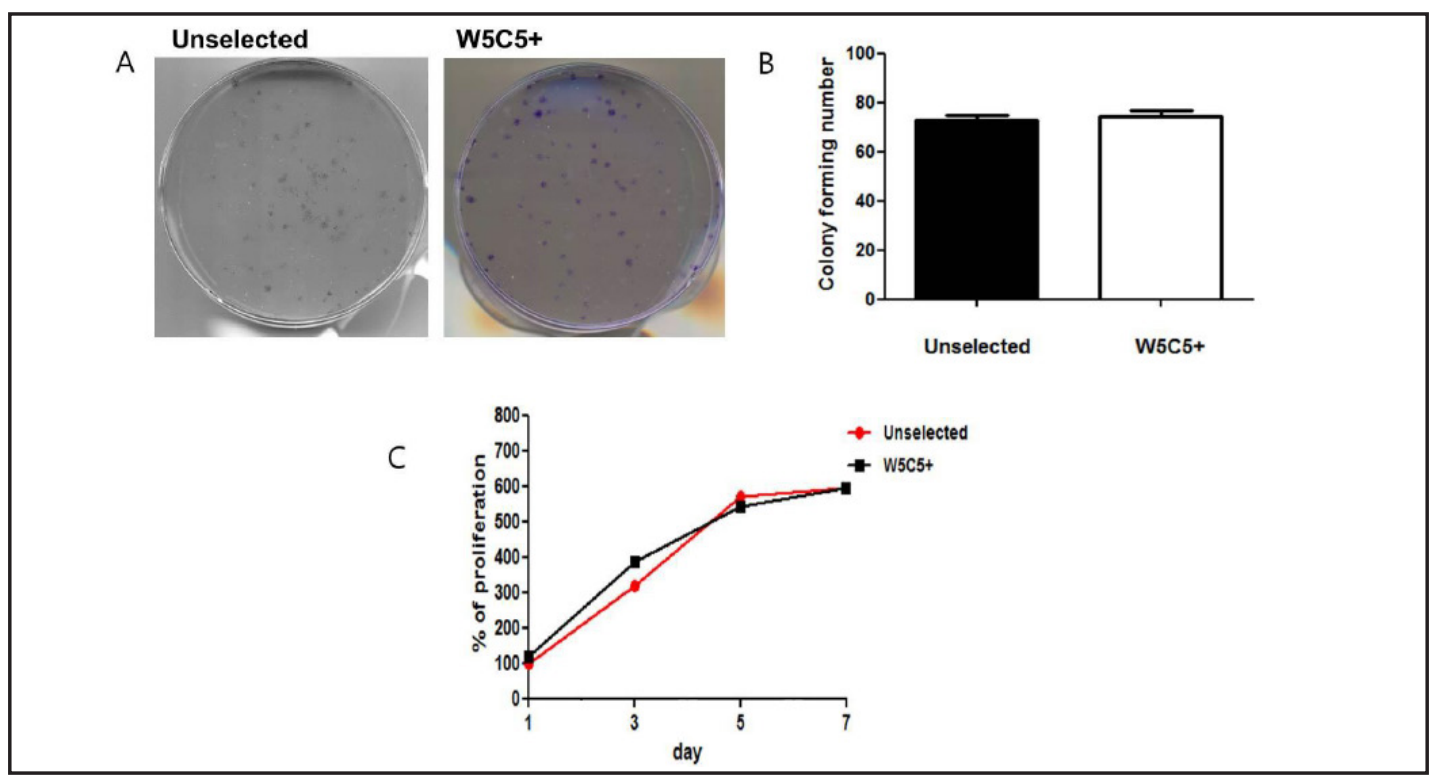

Fig. 2. Proliferative assay on W5C5+ cells and unselected tonsil stromal cells. (A) CFU on W5C5+ cells and unselected T-MSCs. (B) The number of CFUs of W5C5+ cells was not significantly different from that of unselected cells. (C) The proliferative abilities of W5C5+ cells and unselected cells were similar in the MTT assay.

typical MSC phenotypic markers using flow cytometry. W5C5 expression in W5C5+ cells $(20.3 \pm 2.5)$ increased significantly compared to that in unselected T-MSCs $(2.2 \pm 0.8)$. Isolated W5C5+ cells and unselected T-MSCs were positive for expression of marker CD 90, CD44, and CD73 but negative for CD31, CD11b, and HLA-DR in a similar pattern. A representative flow histogram is shown in Fig. 3 (Table 1). In addition, the expression levels of other stemness markers of MSCs-such as STRO-1, CD271, SSEA-4, and CD146-were similar in W5C5+ cells and unselected T-MSCs. However, CD105 expression in W5C5+ cells (53.2 \pm 2.2$)$ was significantly decreased compared to unselected T-MSCs (96.6 \pm 0.4 ).

Comparison of differentiation of human palatine tonsil W5C5+ and unselected cells

We investigated the differentiation potential of W5C5+ cells and unselected T-MSCs. The differentiation of W5C $5+$ cells into osteoblasts was confirmed by positive Alizarin Red $\mathrm{S}$ staining. There were no significant differences in Alizarin Red S staining of W5C5+ cells compared with unselected T-MSCs (Fig. 4-A). The mRNA expression levels of osteocalcin and ALP in W5C5+ cells and unselected T-MSCs did not differ significantly (Fig. 4-A). Morphologically, the formation of cytoplasmic lipid droplets was observed in W5C5+ cells after adipogenic induction, as indicated by Oil Red 0 staining. There were no significant differences in the Oil Red 0 staining of T-MSCs compared with unselected T-MSCs (Fig. 4-B). The expression levels of LPL and PPAR $\gamma$ mRNA in W5C5+ cells and unselected T-MSCs also did not differ significantly (Fig. 4-B). The chondrogenic differentiation potential was assessed, as described above. No apparent differences in shape or size were observed in the pellets of the chondrocytes differentiated from W5C5+ cells or unselected T-MSCs (Fig. 4-C). The mRNA expression levels of AGN and COL2A1 in the two types of cells did not differ (Fig. $4-\mathrm{C})$.

Immunohistochemistry and immunocytochemistry of W5C5+ cells

We investigated the location of the W5C5+ cells in tonsillar tissues by immunohistochemistry. Blood vessels (green color) located between lymphoid follicles were stained with CD31 (a marker expressed in all endothelial cells). W5C5+ cells (red color) were identified in the perivascular area around CD31-positive blood vessels (Fig. 5-A). W5C5+ perivascular cells did not express CD31 marker. 


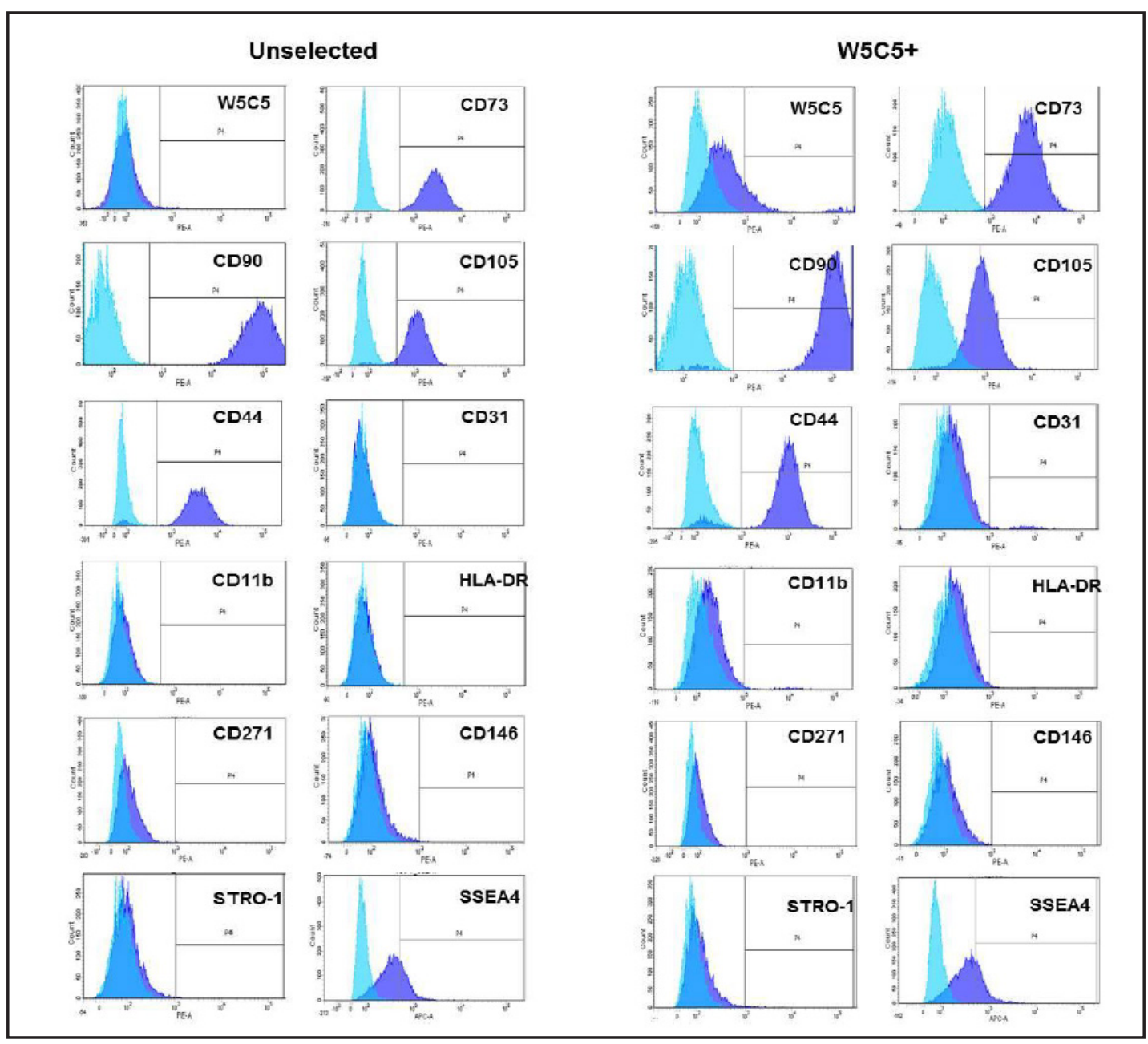

Fig. 3. Surface phenotype on W5C5+ cells and unselected tonsil stromal cells. W5C5+ cells were examined for the expression of lineage markers and typical MSC phenotypic markers using flow cytometry. Isolated W5C5+ cells and unselected T-MSCs were positive for expression of marker CD 90, CD44, and CD73 but negative for CD31, CD11b, and HLA-DR in a similar pattern. The expression of STRO-1, CD271, SSEA-4, and CD146 were similar in W5C5+ cells and unselected T-MSCs. However, CD105 expression in W5C5+ cells was significantly decreased compared to that in unselected T-MSCs.

Fluorescent immunocytochemical staining showed that W5C5 antigen was expressed in the cytoplasm of W5C5+ cells (Fig. 5-B). The expression of W5C5 antigen was significantly higher in W5C5+ T-MSCs $(21.4 \pm 1.8)$ compared to unselected cells $(2.1 \pm 1.4$ ) (Fig. $5-C$ ).

\section{Discussion}

The isolation of purified MSCs from heterogeneous cell populations of MSCs is important to obtain consistent and effective clinical results in the cell-based therapy and tissue engineering field. Recent studies were performed to identify purified MSCs using
Table 1. Surface marker expression of W5C5+ tonsil stromal cells

\begin{tabular}{lccc}
\hline & W5C5+ cells & Unselected cells & P-value \\
\hline W5C5 & $20.3 \pm 2.5$ & $2.2 \pm 0.8$ & $\mathrm{P}<0.05$ \\
CD73 & $99.4 \pm 02$ & $99.2 \pm 0.3$ & NS \\
CD90 & $99.9 \pm 0.1$ & $99.5 \pm 0.2$ & NS \\
CD105 & $53.2 \pm 2.2$ & $96.6 \pm 0.4$ & $\mathrm{P}<0.05$ \\
CD44 & $93.3 \pm 1.2$ & $92.2 \pm 0.4$ & NS \\
CD31 & $1.1 \pm 0.5$ & $1.5 \pm 0.2$ & NS \\
CD11b & $0.4 \pm 0.2$ & $0.8 \pm 0.1$ & NS \\
HLA-DR & $0.1 \pm 0.2$ & $0.5 \pm 0.1$ & NS \\
CD271 & $0.7 \pm 0.4$ & $0.8 \pm 0.5$ & NS \\
CD146 & $0.3 \pm 0.2$ & $0.4 \pm 0.4$ & NS \\
STRO-1 & $0.8 \pm 0.4$ & $0.7 \pm 0.5$ & NS \\
SSEA4 & $20.1 \pm 0.2$ & $19.8 \pm 0.1$ & NS \\
\hline
\end{tabular}




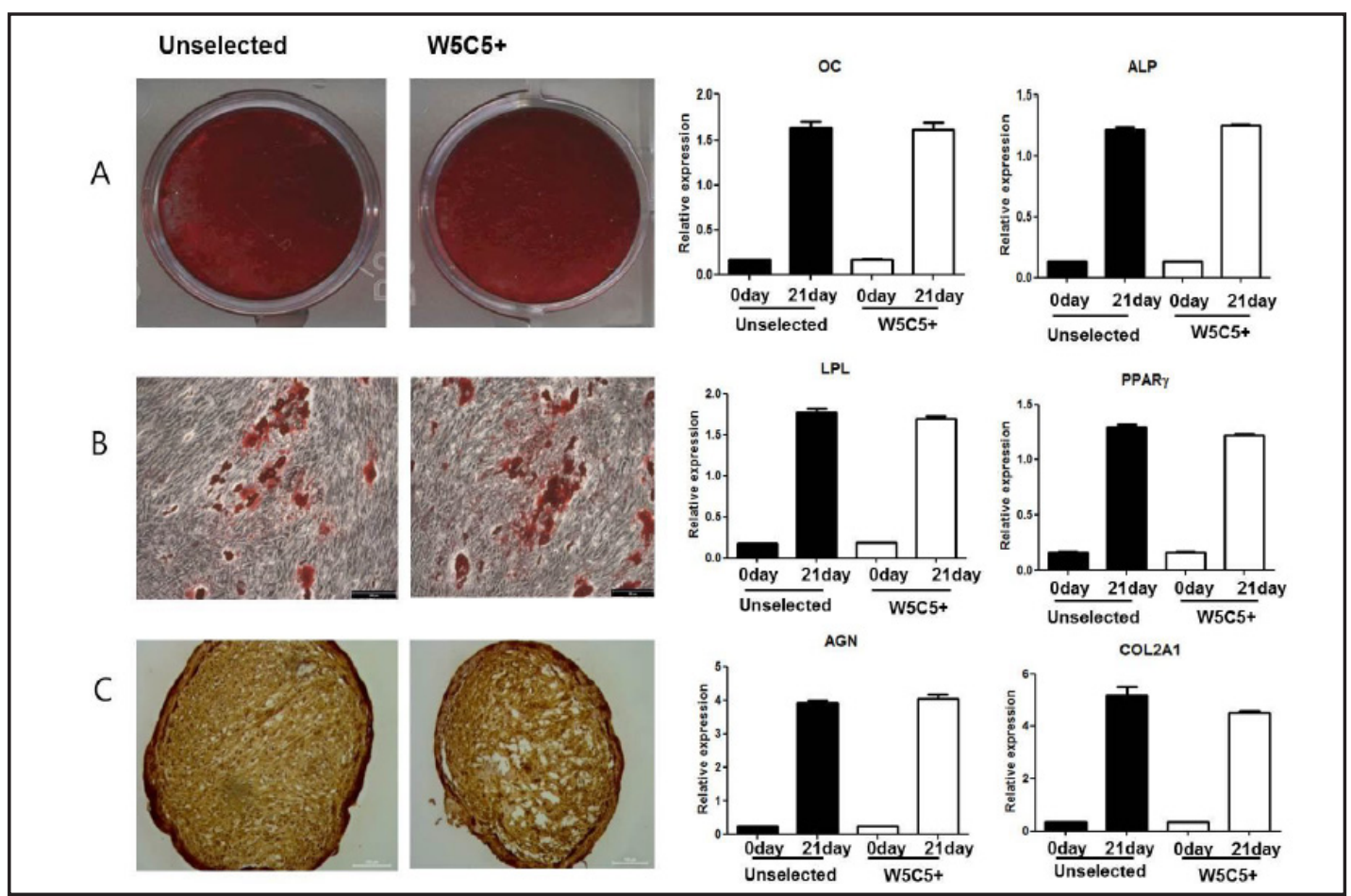

Fig. 4. Differentiation potential on W5C5+ T-MSCs and unselected T-MSCs. (A) Osteogenic differentiation potential. There were no significant differences in Alizarin Red S staining of W5C5+ T-MSCs and unselected T-MSCs. The mRNA expression of OC and ALP did not differ significantly after osteogenic induction of W5C5+ T-MSCs and unselected T-MSCs. (B) Adipogenic differentiation potential. Lipid droplets were stained with Oil Red O. There were no significant differences in Oil Red 0 staining of W5C5+ T-MSCs and unselected T-MSCs. The expression of LPL and PPAR $\gamma$ mRNA did not differ significantly after adipogenic induction of W5C5+ T-MSCs and unselected T-MSCs. (C) Chondrogenic differentiation potential. The sulfated glycosaminoglycan-rich matrix was stained by immunohistochemistry for collagen type-II $\alpha 1$. There were no significant differences in the collagen type-II $\alpha 1$ staining of W5C5+ T-MSCs and unselected T-MSCs. The mRNA expression of AGN and COL2A1 did not differ significantly after adipogenic induction of W5C5+ T-MSCs and unselected T-MSCs. The vertical axis of the graph shows relative gene expression levels. Gene expression levels are presented as relative expressions over glyceraldehyde-3-phosphate dehydrogenase (GAPDH). Specific transcript levels of marker genes were normalized by comparison to those of GAPDH. Data represent means \pm SEM of five different experiments.

specific surface markers from various tissues (e.g., BM, adipose, endometrial, placental, umbilical cord) $[13,18]$. Single markers or combinations of various markers (e.g., STRO1, CD271, SSEA-4, CD146, CD349, CD271(+)SSEA-4(+), CD271(+)CD146(+), or W5C5) have been used to purify MSCs $[13,18]$. Although T-MSCs have been studied as a new autologous or homologous source of MSCs, research on specific markers of the MSCs for purified T-MSC isolation and the localization of MSCs using these markers has not yet been reported.

W5C5, a known epitope of the sushi domain containing two (SUSD2) molecules, was recently identified as the transmembrane protein of 820 amino acids consisting of a large extracellular region containing a Somatomedin B, an adhesion-associated domain in MUC4 and other proteins, a von Willebrand factor, and a Sushi domain [3]. Sushi domains are components of a variety of complement and adhesion proteins. In a recent study, SUSD2 was reported as being differentially expressed in skeletal muscle during the development of obesity [19]. In addition, SUSD2 was shown to be a novel protein suppressing tumorigenic phenotypes, as inhibition of anchorage-dependent and anchorage-independent growth, motility, and invasiveness of cancer cells in culture [20].

\section{KARGER}




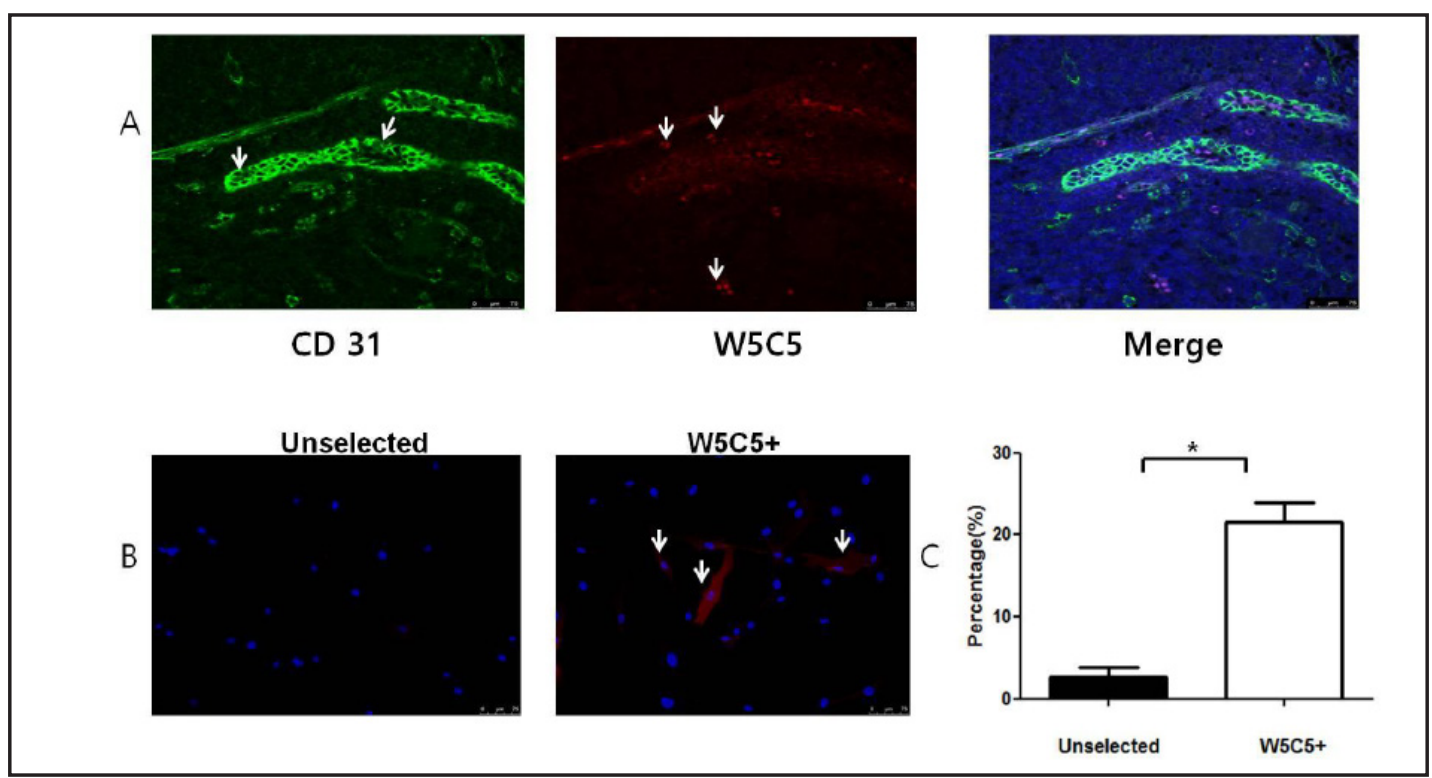

Fig. 5. Immunohistochemistry and immunocytochemistry of W5C5+ cells. (A) Immunohistochemistry of W5C5+ cells. Human tonsil paraffin sections were stained with W5C5 and CD31. White arrows indicate blood vessels with CD31+ (green color). W5C5+ cells (red color) were identified in the perivascular area around CD31+ blood vessels. Nuclei were stained with DAPI. (B) Immunocytochemistry of W5C5+ cells. W5C5 antigen (red color) was expressed in the cytoplasm of W5C5+ cells (white arrows). (C) The expression of W5C5 antigen was significantly higher in W5C5+ T-MSCs compared to that in unselected cells.

W5C5 expressed in the basal and functional layer of the endometrium, and it has usually been studied as a novel single marker for purifying endometrial MSCs [16, 21]. W5C5 could be used as a novel marker for the prospective isolation of endometrial MSCs from biopsy, curettage, or hysterectomy tissue. In addition, W5C5 expressed with high selectivity on human bone marrow-derived MSCs [3]. In the present study, W5C5 was identified as a novel single marker for purifying T-MSCs, which self-renew and differentiate into adipogenic, osteogenic, and chondrogenic cell lineages. W5C5+ cells expressed in the perivascular area of the tonsil tissue.

W5C 5 cells were expressed in $4.2 \pm 0.6 \%$ of endometrial stromal cells [16]. The cloning efficiency of endometrial W5C5+ cells was significantly higher than that of unselected cells and W5C5- cells of endometrial stromal cells. Although W5C5 expression in tonsil stromal cells $(2.5 \pm 0.4 \%)$ was lower than that in endometrial stromal cells $(4.2 \pm 0.6 \%)$, the cloning efficiency of tonsil W5C5+ cells was significantly higher than that of unselected cells and W5C5- cells and similar to that of endometrial W5C5+ cells. Freshly isolated tonsil stromal W5C5+ cells expressed typical MSC markers, including CD73, CD90, and CD44 but not CD11b, CD34, or HLA-DR. Although the expression levels of SSEA-4 in tonsil W5C5+ cells and unselected T-MSCs were relatively high, there was no significant difference between the two types of cells. Most of the clonogenic cells presented in the tonsil stromal cells could select using W5C5. The advantage of purified MSCs selection by W5C5 marker is that will produce many more cells upon culture expansion. W5C5 could also be used as a novel marker for the prospective isolation of T-MSCs from biopsy or tonsillectomy tissue.

Endometrial stromal W5C5+ cells differentiated into adipocytes, osteocytes, chondrocytes, myocytes, and endothelial cells. Masuda et al. reported that the in vitro differentiation potential of endometrial stromal W5C5+ cells was similar to that of BM-MSCs. Sivasubramaniyan et al. reported that only SUSD2+, but not SUSD2-, BM cells gave rise to colony-forming units-fibroblasts and were able to differentiate into osteoblasts, adipocytes, and chondrocytes [3]. The present results show that tonsil stromal W5C5+ cells also showed a multipotent differentiation ability in vitro, similar to that of unselected T-MSCs. In addition, 
W5C5 + cells were meeting the criteria of the MSC with clonogenecity, surface markers, and multipotent differentiation ability.

Some specialized niches store and maintain stem cells and mediate the balanced response of MSCs to the needs of the organism. Although MSCs that have self-renewal and differentiation potential have been identified in a number of in vitro studies, the true identity of MSCs and their niches in vivo is still under debate. MSCs are known to be derived from fibroblasts or perivascular cells of the tissue, but these hypotheses remain controversial. To date, there have been no studies on the location of the niches of isolated T-MSCs in vivo.

Histologically, the tonsils consist of a number of lymphoid follicles involved in the proliferation of lymphocytes. Lymphatic ducts, blood vessels, and tonsillar crypts exist between lymphoid follicles. In this study, W5C5+ cells were observed in the perivascular area of the blood vessel between the lymphoid follicles in the tonsil. These findings are similar to those indicating that W5C5+ cells are found in the perivascular area of the basal and functional layer of the endometrium [17]. Several studies have shown that in vivo niches of MSCs are located in the pericytes or perivascular areas of various tissues or organs [13,22]. Similar to MSCs, pericytes and perivascular cells are able to differentiate into osteoblasts, chondrocytes, adipocytes, fibroblasts, myofibroblasts, and smooth muscle cells in vitro. Our results suggest that niches of T-MSCs are also located in the perivascular areas of tonsil tissues. These findings on the localization of T-MSCs are consistent with those of other research on the niches of MSCs.

There have been many attempts to apply MSCs in various clinical fields. Although there has been a lot of research on BM as a source of MSCs, the tonsils are being studied as a new source of MSCs because tonsil tissues are easily harvested under local anesthesia and the donors of T-MSCs are relatively young. The identification of purified MSCs from a heterogeneous population of MSCs is an important process for the clinical application of MSCs. Therefore, in this study, W5C5 was used for the isolation of purified T-MSCs. Our study confirmed that W5C5+ T-MSCs from tonsil stromal cells had a high CFU enrichment capacity and the typical characteristics of MSCs (e.g., similar proliferative ability, surface phenotypes, and differentiation potential), and that W5C5+ cells were located in the perivascular areas of the tonsil tissues. In future, studies on the functional role of W5C5 in MSCs will be needed.

In conclusion, our study showed that W5C5+ T-MSCs possessed typical MSC properties with high colony-forming efficiency and that niches of W5C5+ T-MSCs were located in the perivascular areas of tonsil tissues. These findings suggest that W5C5 is a useful single marker for the isolation of purified T-MSCs.

\section{Acknowledgments}

This work was supported by the Basic Science Research Program through a National Research Foundation of Korea (NRF) grant funded by the Ministry of Education, Science, and Technology (No. NRF 2014R1A2A1A11052999).

\section{Disclosure Statement}

The authors report no conflicts of interest. The authors alone are responsible for the content and writing of the paper

\section{References}

1 Pittenger MF, Mackay AM, Beck SC, Jaiswal RK, Douglas R, Mosca JD, Moorman MA, Simonetti DW, Craig S, Marshak DR: Multilineage potential of adult human mesenchymal stem cells. Science 1999;284:143-147. 


\section{Cellular Physiology Cell Physiol Biochem 2016;38:83-93 \begin{tabular}{l|l|l} 
and Biochemistry 10.1159/000438611 & $\begin{array}{l}\text { C) } 2016 \text { The Author(s). Published by S. Karger AG, Basel } \\
\text { www.karger.com/cpb }\end{array}$ \\
\hline
\end{tabular}}

Lee et al.: Isolation and Localization of T-MSCs by W5C5

2 Manuguerra-Gagne R, Boulos PR, Ammar A, Leblond FA, Krosl G, Pichette V, Lesk MR, Roy DC:

Transplantation of mesenchymal stem cells promotes tissue regeneration in a glaucoma model through laser-induced paracrine factor secretion and progenitor cell recruitment. Stem Cells 2013;31:1136-1148.

3 Sivasubramaniyan K, Harichandan A, Schumann S, Sobiesiak M, Lengerke C, Maurer A, Kalbacher H, Buhring HJ: Prospective isolation of mesenchymal stem cells from human bone marrow using novel antibodies directed against sushi domain containing 2. Stem Cells Dev 2013;22:1944-1954.

4 Lee BJ, Wang SG, Lee JC, Jung JS, Bae YC, Jeong HJ, Kim HW, Lorenz RR: The prevention of vocal fold scarring using autologous adipose tissue-derived stromal cells. Cells Tissues Organs 2006;184:198-204.

5 Noth U, Steinert AF, Tuan RS: Technology insight: Adult mesenchymal stem cells for osteoarthritis therapy. Nat Clin Pract Rheumatol 2008;4:371-380.

6 Eirin A, Zhu XY, Krier JD, Tang H, Jordan KL, Grande JP, Lerman A, Textor SC, Lerman LO: Adipose tissuederived mesenchymal stem cells improve revascularization outcomes to restore renal function in swine atherosclerotic renal artery stenosis. Stem Cells 2012;30:1030-1041.

7 Fukuchi Y, Nakajima H, Sugiyama D, Hirose I, Kitamura T, Tsuji K: Human placenta-derived cells have mesenchymal stem/progenitor cell potential. Stem Cells 2004;22:649-658.

8 Huang GT, Gronthos S, Shi S: Mesenchymal stem cells derived from dental tissues vs. Those from other sources: Their biology and role in regenerative medicine. J Dent Res 2009;88:792-806.

9 Schwab KE, Hutchinson P, Gargett CE: Identification of surface markers for prospective isolation of human endometrial stromal colony-forming cells. Hum Reprod 2008;23:934-943.

10 Sarugaser R, Lickorish D, Baksh D, Hosseini MM, Davies JE: Human umbilical cord perivascular (hucpv) cells: A source of mesenchymal progenitors. Stem Cells 2005;23:220-229.

11 Choi JS, Lee BJ, Park HY, Song JS, Shin SC, Lee JC, Wang SG, Jung JS: Effects of donor age, long-term passage culture, and cryopreservation on tonsil-derived mesenchymal stem cells. Cell Physiol Biochem 2015;36:8599.

12 Lee YS, Lee JE, Park HY, Lim YS, Lee JC, Wang SG, Lee BJ: Isolation of mesenchymal stromal cells (mscs) from human adenoid tissue. Cell Physiol Biochem 2013;31:513-524.

13 Lv FJ, Tuan RS, Cheung KM, Leung VY: Concise review: The surface markers and identity of human mesenchymal stem cells. Stem Cells 2014;32:1408-1419.

14 Ho AD, Wagner W, Franke W: Heterogeneity of mesenchymal stromal cell preparations. Cytotherapy 2008;10:320-330.

15 Ryu KH, Cho KA, Park HS, Kim JY, Woo SY, Jo I, Choi YH, Park YM, Jung SC, Chung SM, Choi BO, Kim HS: Tonsil-derived mesenchymal stromal cells: Evaluation of biologic, immunologic and genetic factors for successful banking. Cytotherapy 2012;14:1193-1202.

16 Masuda H, Anwar SS, Buhring HJ, Rao JR, Gargett CE: A novel marker of human endometrial mesenchymal stem-like cells. Cell Transplant 2012;21:2201-2214.

17 Ulrich D, Tan KS, Deane J, Schwab K, Cheong A, Rosamilia A, Gargett CE: Mesenchymal stem/stromal cells in post-menopausal endometrium. Hum Reprod 2014;29:1895-1905.

18 Calloni R, Cordero EA, Henriques JA, Bonatto D: Reviewing and updating the major molecular markers for stem cells. Stem Cells Dev 2013;22:1455-1476.

19 Bolton K, Segal D, McMillan J, Sanigorski A, Collier G, Walder K: Identification of secreted proteins associated with obesity and type 2 diabetes in psammomys obesus. Int J Obes (Lond) 2009;33:1153-1165.

20 Sugahara T, Yamashita Y, Shinomi M, Yamanoha B, Iseki H, Takeda A, Okazaki Y, Hayashizaki Y, Kawai K, Suemizu H, Andoh T: Isolation of a novel mouse gene, msvs-1/susd2, reversing tumorigenic phenotypes of cancer cells in vitro. Cancer Sci 2007;98:900-908.

21 Buhring HJ, Battula VL, Treml S, Schewe B, Kanz L, Vogel W: Novel markers for the prospective isolation of human msc. Ann N Y Acad Sci 2007;1106:262-271.

22 Crisan M, Yap S, Casteilla L, Chen CW, Corselli M, Park TS, Andriolo G, Sun B, Zheng B, Zhang L, Norotte C, Teng PN, Traas J, Schugar R, Deasy BM, Badylak S, Buhring HJ, Giacobino JP, Lazzari L, Huard J, Peault B: A perivascular origin for mesenchymal stem cells in multiple human organs. Cell Stem Cell 2008;3:301-313. 